\title{
A Nanometric Probe of the Local Proton Concentration in Microtubule-Based Biophysical Systems
}

Aarat P. Kalra a , Boden B. Eakins ${ }^{\text {g }}$, Sergei I. Vagin ${ }^{c}$, Hui Wang ${ }^{b}$, Sahil D. Patel ${ }^{\mathrm{d}}$, Philip Winter ${ }^{\mathrm{e}}$, Maral Aminpour $^{\text {b, e }}{ }^{\text {, John D. Lewis }}{ }^{\text {e, Vahid Rezania }}{ }^{\text {, }}$, Karthik Shankar ${ }^{\mathrm{g}}$, Gregory D. Scholes ${ }^{\text {a }}$, Jack A.

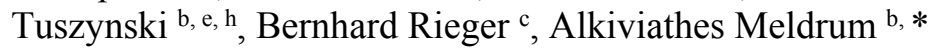

\section{Supporting Information}

a

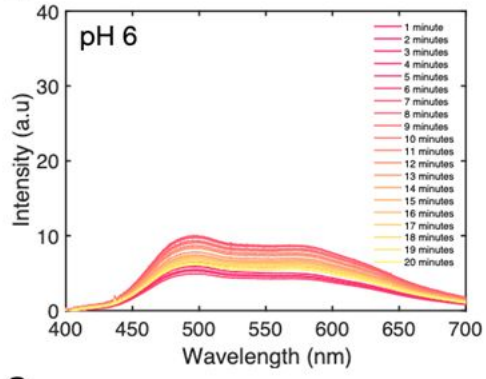

C

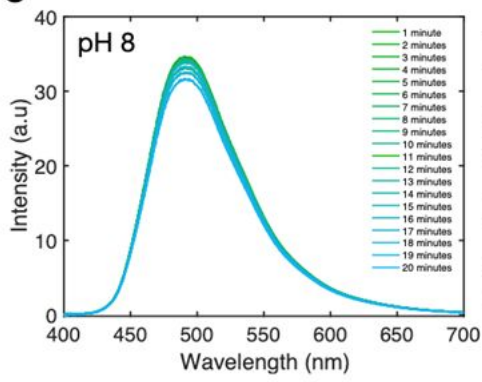

b

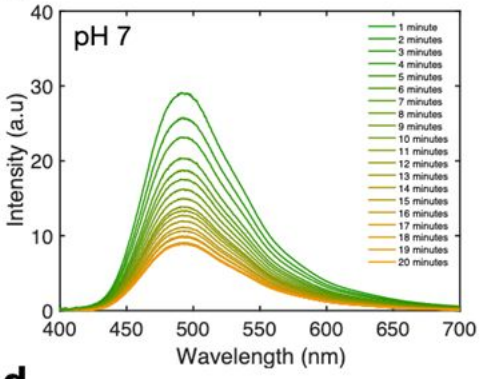

d

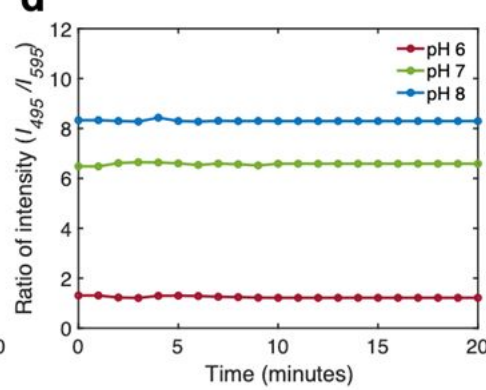

Fig. S1. Fluorescence spectra for various pump laser power in $\mathrm{pH} 6$ (a), (b) $\mathrm{pH} 7$ (b) and $\mathrm{pH} 8$ solutions. Panel (d) shows the measured signal as a function of time. The signal is not noticeably affected by photobleaching. 


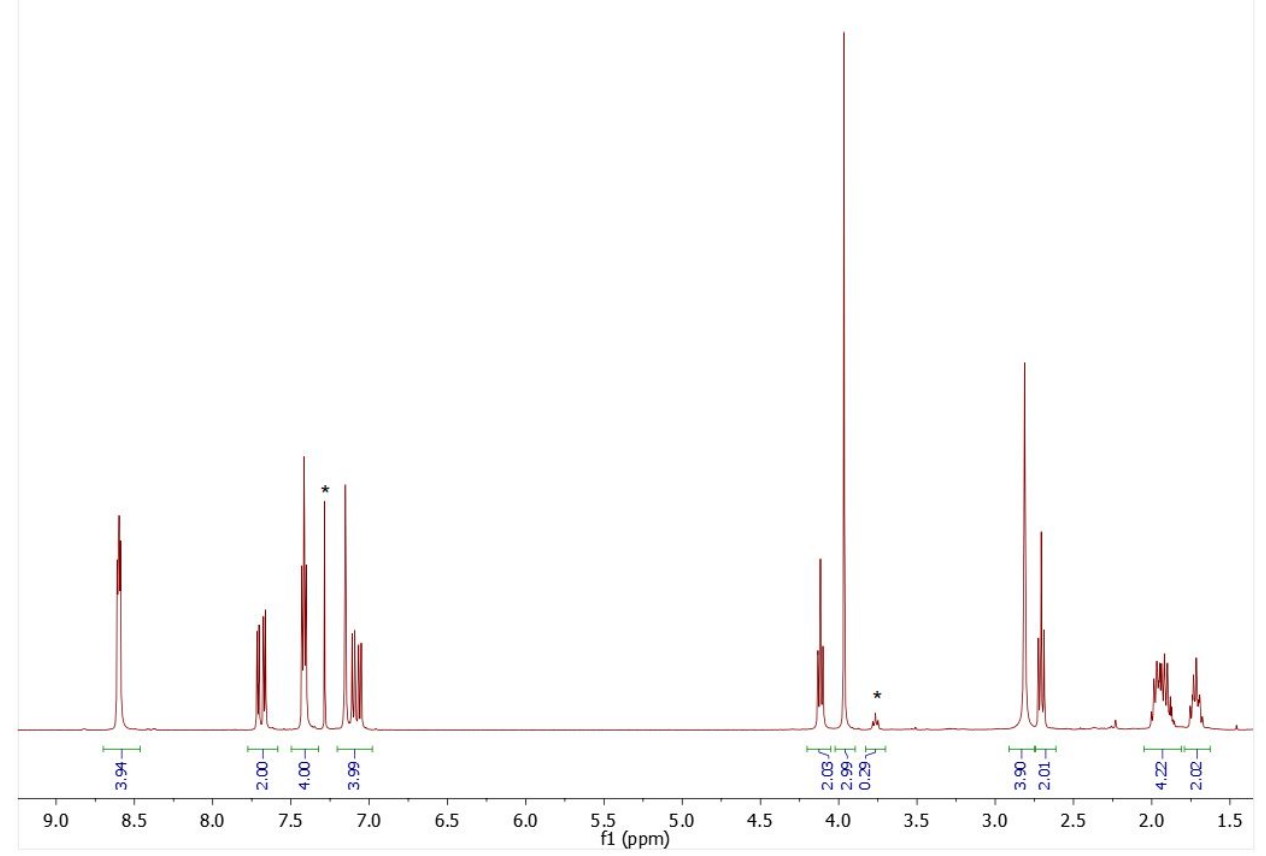

Fig. S2. $1 \mathrm{H}$ NMR spectrum (400 MHz) of NHS-P4VB in $\mathrm{CDCl}_{3}$. The deuterated chloroform (7.26 ppm) and residual THF (3.75 ppm) signals are marked with asterisk. The second THF peak is beneath the multiplet at $1.9 \mathrm{ppm}$.

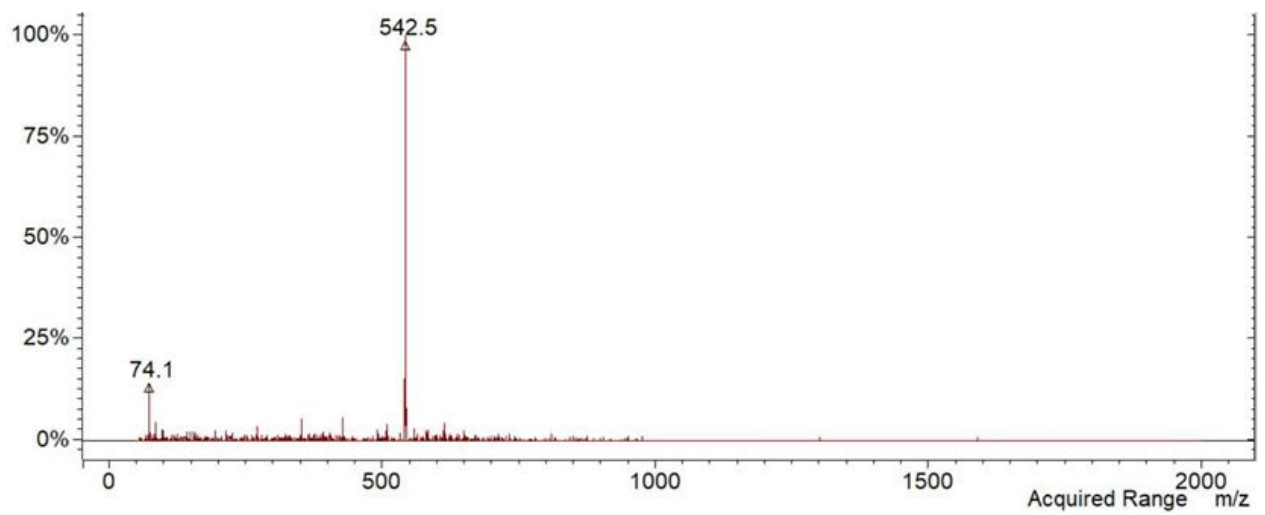

Fig. S3. Electrospray ionization mass spectroscopy (ESI-MS) spectrum of NHS-c-P4VB (positive mode), recorded in acetonitrile with a Varian LC MS 500 instrument (direct injection). 


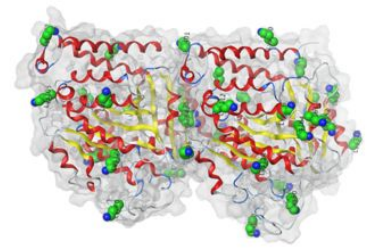

b
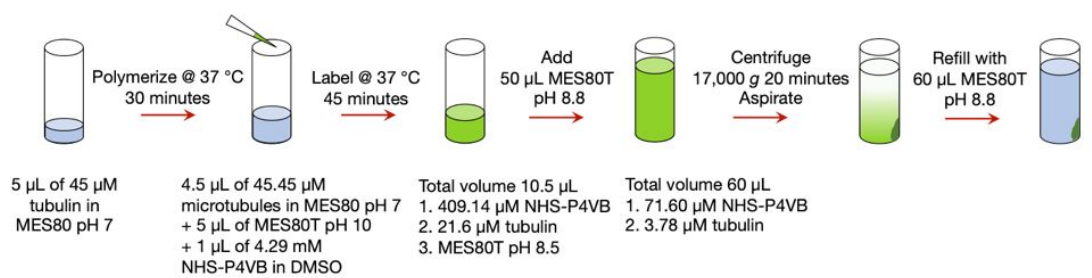

Fig. S4. (a) Diagram illustrating the structure of $\alpha, \beta$ tubulin with lysine residues marked in green. The Ctermini do not contain any lysine residues and have hence been left out of the plot. (b) Schematic of the protocol for the microtubule synthesis and labeling.

a

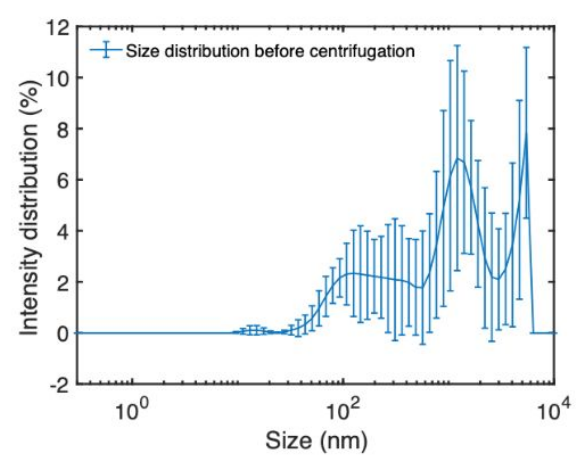

b

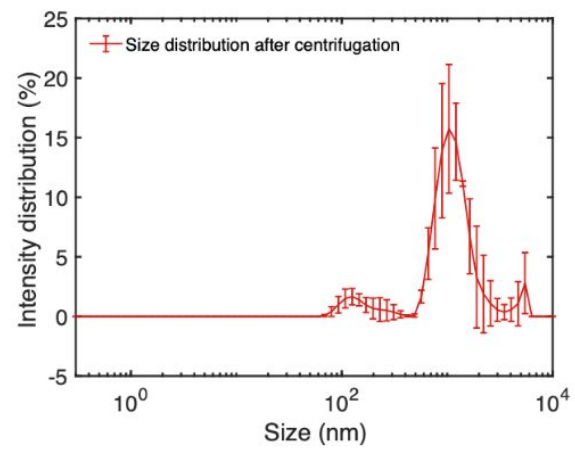

Fig. S5. (a) A DLS size distribution profile of $3.7 \mu \mathrm{M}$ tubulin solution before centrifugation, containing unpolymerized tubulin, microtubules and free P4VB. The large variation and noise in the particle size indicates a high polydispersity index. (b) A DLS size distribution profile of $2.2 \mu \mathrm{M}$ tubulin solution after centrifugation, aspiration and re-introduction of MES80T at $\mathrm{pH} 7$. The smaller particles were clearly removed by this step. 
a

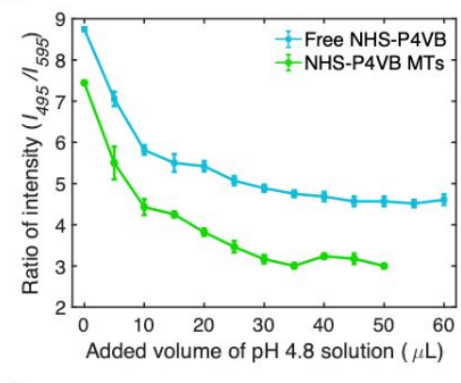

C

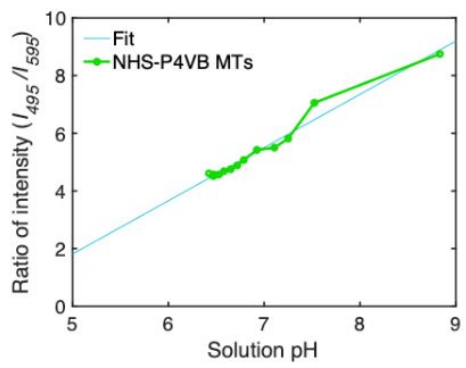

b

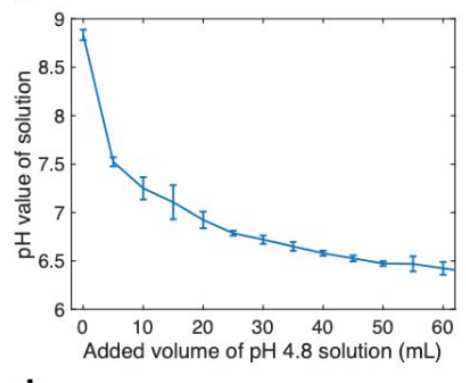

d

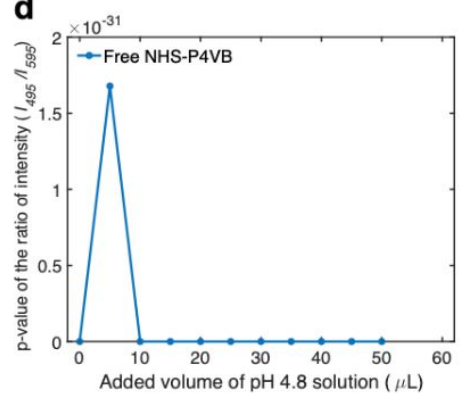

Fig. S6. Errors and uncertainties. (a) represents the sensorgrams in which each point is the average over the step time window and the error bars show the measured standard deviation. (b) shows the measured $\mathrm{pH}$ as a function of added volume of $\mathrm{pH} 4.8$ buffer solution to the stock $\mathrm{pH} 8$ solution. To make these measurements, the volumes were scaled up by a factor of 1000 . The error bars represent the standard deviation over three independent measurements at each concentration. In Fig. 3 the errors were obtained from six individually prepared solutions of the chromophore in BRB80 $\mathrm{pH} 7$ solution. Part (c) shows the fluorescence signal as a function of the measured $\mathrm{pH}$, with a linear calibration fit to the data. This was used to calibration the NHS-P4VB signal response. (d) The variation of p-value evaluated from a two-sample ttest as a function of added volume of $\mathrm{pH} 4.8$ buffer. The low values indicate that the microtubule data (local $\mathrm{pH}$ ) is different from the solution data (solution $\mathrm{pH}$ ) with a very high degree of certainty. The "spike" at the $5 \mu \mathrm{L}$ volume is due to the larger error bar for that measurement, as shown in panel (b).

There is a low probability process of protons weakly attached to tubulin in solution being transferred to the P4VB molecule bound to its surface. This cannot be excluded a priori but its likelihood is very low, because the only residue that can be a source of removable protons is histidine. Histidine's pKa is 6.0 and its imidazole ring is mostly protonated below $\mathrm{pH} 6$ while above $\mathrm{pH} 6$ this proton is lost. For it to become transferred to $\mathrm{P} 4 \mathrm{VB}$, one of the histidine residues needs to be within $\sim 1 \mathrm{~nm}$ distance to the location of $\mathrm{P} 4 \mathrm{VB}$, which binds to lysine. In total there are 5 Lys residues on alpha tubulin and 3 Lys residues on beta tubulin, which are close enough to His residues. The residue numbers in red are the ones that are close to a HIS residue. Below we list a summary of the shortest distances between CB of LYS residues and the center of mass of LYS with the histidines in their vicinity (less than $10 \mathrm{~A}$ ) for the alpha tubulin monomer followed by the beta tubulin monomer. This is illustrated graphically in Fig. S7. These results were obtained using a tcl script in VMD software to calculate them. Furthermore, previous simulations of this molecule using density functional theory [Thungon et al., in review] showed that the P4VB pyridine groups are much less favorable sites for protons, as compared to carboxylic groups. Finally, the MTs might provide a local effect not involving a proton such as solvatochromism; however, we find that the P4VB spectral shifts of the type observed relies on the availability of a proton or a few other cations such as $\mathrm{Zn}^{2+}$ and $\mathrm{Hg}^{2+}$. For all these reasons, we expect that the likelihood of interferences arising from protons on the MTs themselves is highly unlikely.

\section{LYS residue numbers- chain A (alpha tubulin)}


112124163164166280304311326336338352370394404014306096

HIS residue numbers chain A (alpha tubulin)

1071391921972662828330939340661888

Lys residues close to His in Chain A (alpha tubulin)

The distances in Angstroms between these residues are given below

HIS107:CB/LYS112:CB = 7.625906

Com: 6.988321799414744

LYS166:CB/HIS197:CB $=6.360817$

com: 7.233380128708278

HIS28:CB/LYS60:CB $=7.858958$

8.430405338119328

HIS309:CB/LYS311: $\mathrm{CB}=7.361284$

Com: 6.118431578708777

HIS393:CB/LYS394: $\mathrm{CB}=5.350030$

Com: 5.452560281439389

LYS60:CB/HIS61:CB $=5.201306$

Com: 5.3335147782586825

$+$

LYS residue numbers - chain B (beta tubulin)

1051241561761921825429932633835237238940260

His residue numbers - chain B (beta tubulin)

10713919222926628309374066

Lys residues close to His in Chain B (beta tubulin)

The distance is in the Angstrom unit

LYS105:CB/HIS107:CB $=7.637268$

Com: 9.271497882645034

LYS19:CB/HIS229:CB $=5.068713$

Com: 6.488138579705152

LYS402:CB/HIS406:CB $=7.692636$

Com: 9.31053134786413 


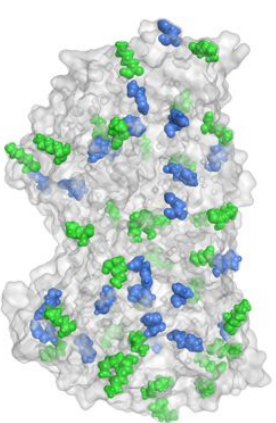

Fig. S7 The tubulin dimer showing histidine (blue) and lysine (green) residues which are located within a $1 \mathrm{~nm}$ distance of each other.

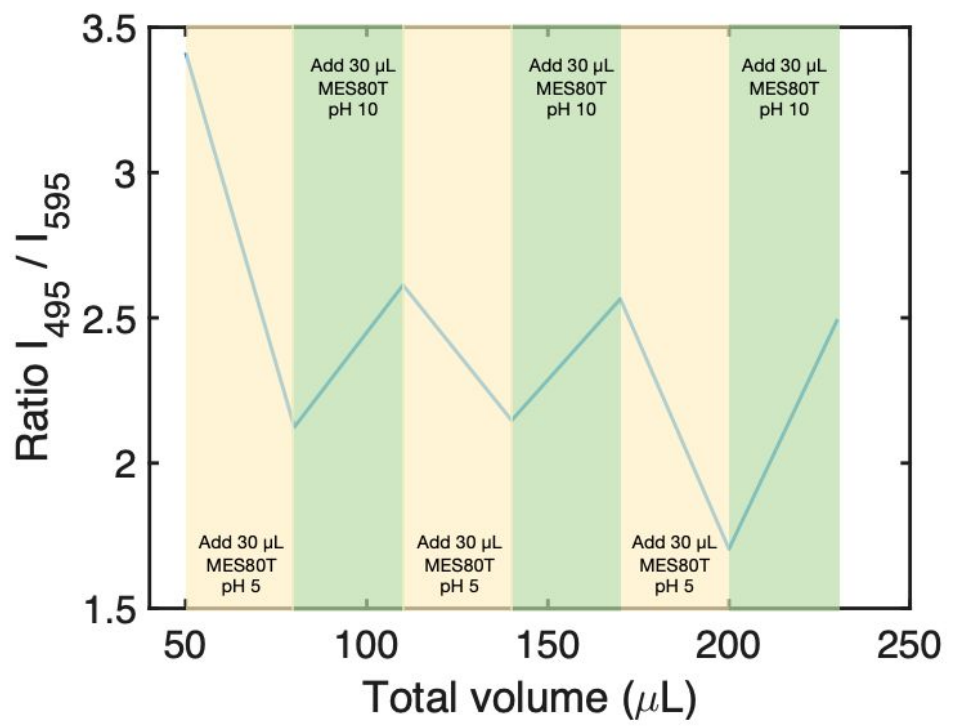

Fig. S8. The reversal in the I495/I595 ratio of NHS-P4VB conjugated microtubules. The initial volume of the solution contained $50 \mu \mathrm{L}$ of MES80T $\mathrm{pH} 9$.
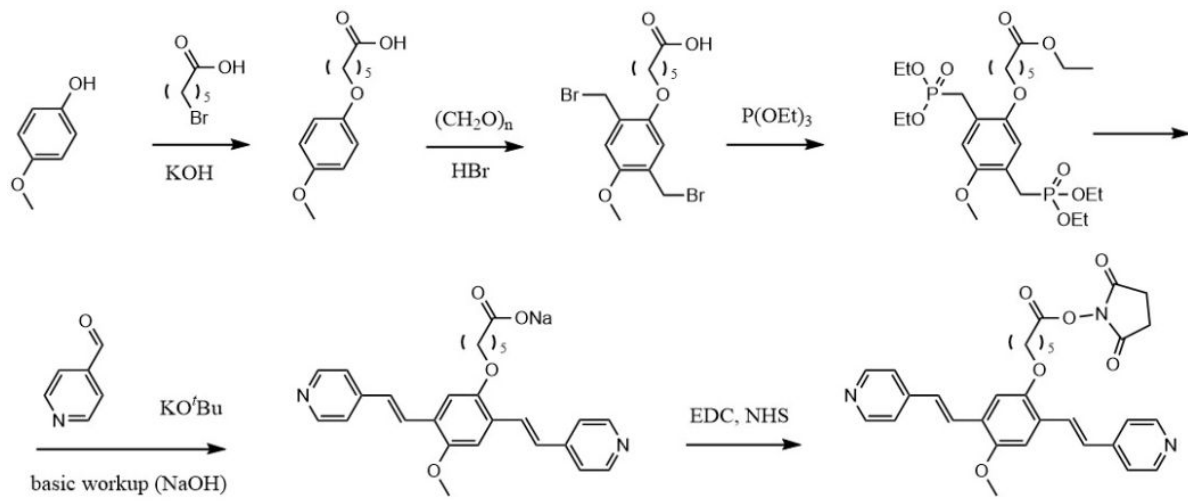

c-P4VB

NHS-P4VB

Fig. S9. Detailed chemical processes for the synthesis of NHS-P4VB. 